\title{
Conceptual methods of starting and controlling operating modes of compressorless combined cycle gas turbine unit
}

\author{
Mikhail Sinkevich ${ }^{1,2}$, Eldar Ramazanov ${ }^{2, *}$, and Anatoliy Kosoy ${ }^{1,3}$ \\ ${ }^{1}$ Joint Institute for High Temperatures of the Russian Academy of Sciences, Izhorskaya St., Bldg. 13, Block 2, Moscow, Russia \\ ${ }^{2}$ Peoples' Friendship University of Russia (RUDN University), 6 Miklukho-Maklaya St, Moscow, 117198, Russian Federation \\ ${ }^{3}$ Moscow Power Engineering Institute, Krasnokazarmennaya str., Bldg. 14, Moscow, Russia
}

\begin{abstract}
A method for operation study of a compressorless combined-cycle gas turbine (CCCGT) unit during transients from one steady-state mode to another, including during start-up and shutdown, is proposed. As an example, studies of the transients of an energy complex with a capacity of $60 \mathrm{MW}$ are given. A variant of an algorithm concept for start/stop and transients of such an energy complex is proposed. This concept includes algorithms for starting from the three initial states of the energy complex (cold, hot and standby). For these algorithms, the time and energy consumption characteristics are estimated. It is shown that from a cold state, such an energy complex can be started and brought to the nominal mode in 1.5-2 hours. At the same time, the energy costs will not exceed energy consumption for its own needs in a nominal mode. The start-up time from the hot state can be reduced to 20-25 minutes, and the start-up from the standby state can be 2.5-3 minutes. The proposed concept of control algorithms for transient modes is such, so that a temperature state of the main components and parts of the energy complex is provided at a constant temperature. Thus, the time of transients will be determined only by the speed of the regulatory authorities. Consequently, power systems operating on the CCCGT cycle can operate equally efficiently, both in the basic mode and in the power control mode.
\end{abstract}

Keywords. Compressorless combined-cycle, start-stop algorithms, transition process, dynamic characteristics

\section{Introduction}

Recently, fundamentally new energy generation cycles are actively considered, which not only provide high thermal efficiency, but also provide minimal environmental impact [1], [2], [3], [4], [5]. Among such cycles, one can distinguish a cycle of a compressorless combined cycle gas turbine (CCCGT) unit [6], [7], [8]. This cycle, while generating electricity and heat together, exceeds the main known cycles in terms of thermal efficiency [9], [10]. However, during a creation process of new energy complexes, it is important to have a concept of a complex operation in non-calculated modes at the very early stages of design. This includes transitioning from one steady-state mode to another, start and stop. If you do not take into account the need to pass these modes, it may turn out that designed energy complex will not achieve required operating modes. The difficulty of studying these modes at the first stages of design is lack of information about the object under research (the work is performed when the complex has not yet been designed and there is no information about its constituent nodes). The objectives of a research are: to analyze possible algorithms for start / stop the power complex and operating in transient modes; to determine time characteristics of start/stop and transients; to formulate the requirements for the designed equipment necessary for the implementation of these operating modes.

\section{Description of a compressorless combined cycle gas turbine unit scheme}

A compressorless combined cycle gas turbine unit scheme is shown in Fig. 1. A detailed description of the CCCGT scheme can be found in [9], [10]. Immediately after the pump, the $\mathrm{CO}_{2}$ enters a $\mathrm{CO}_{2}$ heater, then a $\mathrm{CO}_{2}$ recuperator, and then the combustion chamber. The water before entering the combustion chamber is heated only in a $\mathrm{H}_{2} \mathrm{O}$ recuperator. After the combustion chamber the working fluid is operated in the turbine unit, and then its heat is sequentially recovered back into the cycle in recuperators and a contact condenser. Part of the heat received in contact condensers is sent to the heat network. The thermodynamic cycle is completed in the $\mathrm{CO}_{2}$ liquefaction system, where the heat is removed to the environment, and the $\mathrm{CO}_{2}$ passes into a liquid phase. In working condition, shutoff valves 27-32 are open, 25, 26 are closed, and overflow valves 35,36 are closed. When starting and stopping the power complex, these valves are controlled according to the appropriate algorithms.

\footnotetext{
* Corresponding author: christmas071@ gmail.com
} 


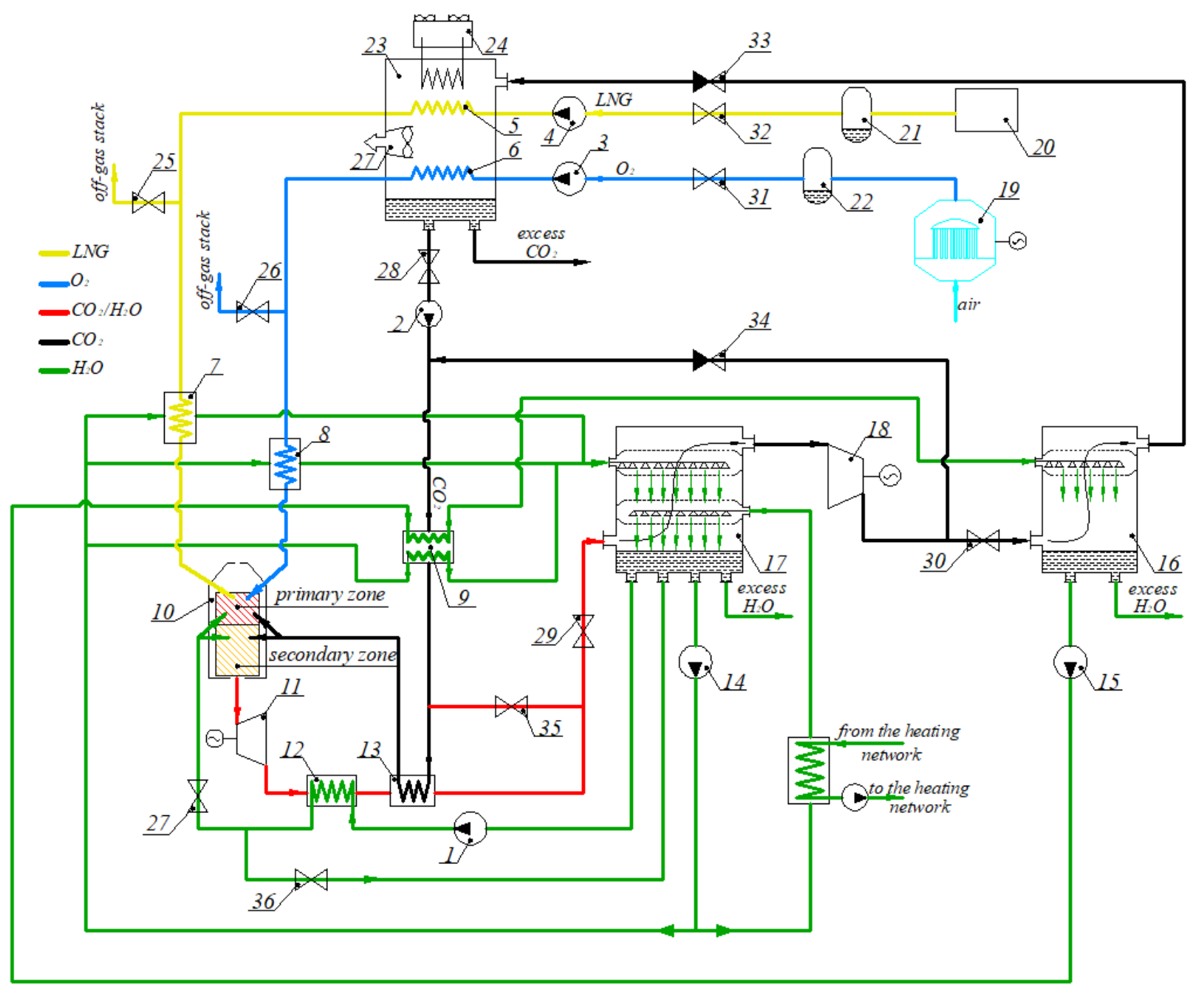

Fig. 1. Scheme of a compressorless combined cycle gas turbine unit

\begin{tabular}{|c|c|c|c|}
\hline Element & Number of position & Element & Number of position \\
\hline Feed pumps of $\mathrm{H}_{2} \mathrm{O}, \mathrm{CO}_{2}, \mathrm{O}_{2}$ and $\mathrm{LNG}$ & $1,2,3$ and 4 & Air separation unit & 19 \\
\hline Cold utilizers of LNG and $\mathrm{O}_{2}$ & 5 and 6 & Fuel handling unit & 20 \\
\hline Heaters of LNG, $\mathrm{CO}_{2}$ and $\mathrm{O}_{2}$ & 7,8 and 9 & $\begin{array}{l}\text { Storage of liquid } \\
\text { LNG and } \mathrm{O}_{2}\end{array}$ & 21 and 22 \\
\hline Combustor camber & 10 & $\begin{array}{l}\mathrm{CO}_{2} \\
\text { system }\end{array}$ & 23 \\
\hline Turbine unit & 11 & Refrigerator & 24 \\
\hline $\mathrm{H}_{2} \mathrm{O}$ and $\mathrm{CO}_{2}$ heat exchanger & 12 and 13 & Shutoff valves & $25-32$ \\
\hline Circulating pump & 14 and 15 & Non-return valves & 33,34 \\
\hline Contact condenser & 16 and 17 & Overflow valves & 35,36 \\
\hline Additive $\mathrm{CO}_{2}$ pump & 18 & & \\
\hline
\end{tabular}

\section{Description of the research method}

To solve this problem, a simplified model of the dynamic characteristics of energy complexes operating on the CCCGT cycle is developed. To do this, all the equipment included in the energy complex was divided into groups. One of the groups was excluded from consideration. This group includes: fuel storage and preparation equipment; refrigeration equipment; liquid oxygen production and storage equipment; all infrastructure for heat transfer to the heating system. The exclusion of this group from consideration is due to the fact that, for one reason or another, it practically does not affect the dynamics of transient processes (for example, there are storage tanks for storing liquid $\mathrm{O}_{2}, \mathrm{CH}_{4}, \mathrm{CO}_{2}$ and $\mathrm{H}_{2} \mathrm{O}$, so that the change in the operating modes of the corresponding infrastructure can be quite slow, and all mismatch of the costs of the components of the working fluid will be compensated by these tanks). The second group includes equipment in which a state temperature changes negligibly little 
over the entire range of steady state modes. This group includes cold utilizers, heaters for the initial components of working fluid, and contact condensers. One of the most important parts in this group of equipment is the external power housing of the turbine unit. This group of equipment is taken into account only when calculation of start and stop process is considered. The third group includes parts and components that operate at high temperatures. It includes parts of the combustion chamber heat pipes, parts of the turbine flow part and heat exchangers. Turbomachines are grouped into a separate group. The description of the temperature state of the second and third equipment groups is based on the differential equation of the thermal balance, which can be expressed as follows

$$
d T / d \tau=1 / C^{*} N_{\Sigma}
$$

where $d T / d \tau$ - time derivative of temperature change, $C-$ heat capacity of described system, $N_{\Sigma}$ - power of supplied energy.

The description of the change in turbomachines' speed is based on a differential equation of mechanical energy balance, which can be expressed:

$$
n * d n / d \tau=1 / J * N_{M}
$$

where $n$-rotor rotation speed, $d n / d \tau$ - time derivative of rotation speed, $J$-rotor moment of inertia, $N_{M}$ - the power of mechanical energy used to change rotor speed.

Preliminary studies of the main components of 60 MW power complexes, which have the greatest impact on dynamic processes, are carried out. As a result of these researches, weight and size parameters, including the heat capacity and moments of inertia, of the selected equipment groups are determined.

\section{Cold start algorithm}

Under the cold state, we will understand such a state of an energy complex, in which all equipment has an ambient temperature. The start of a launch algorithm will be counted from a "START" command, which is given after all the preparatory work is completed. At the time of the "START" command, the pressure in the working circuit is minimal, the circuit is filled with almost pure $\mathrm{CO}_{2}$, shutoff valves $25-32$ and an overflow valve 35 are closed, and the shutoff valve 29 and an overflow valve 36 are open. At the "START" command, the additive $\mathrm{CO}_{2}$ compressor 18 is switched on and switched to the warm-up mode. The filling $\mathrm{CO}_{2}$ circuit begins to circulate in a small circuit (exits the compressor 18, passes through the $\mathrm{CO}_{2}$ heater 9, the $\mathrm{CO}_{2}$ heat exchanger 13 on the side of heated coolant, a combustion chamber 10 , a turbine unit 11 , the $\mathrm{H}_{2} \mathrm{O}$ heat exchanger 12 and $\mathrm{CO}_{2}$ heat exchanger 13 on the side of heating coolant, the low-pressure contact condenser 17 and returns to the inlet of the compressor 18). After the compressor 18 reaches speed of the "WARM-UP" mode, $\mathrm{H}_{2} \mathrm{O}$ feed pump $1, \mathrm{O}_{2}$ feed pump 3 and LNG feed pump 4 are switched on, and the ignition system is switched on in the combustion chamber. After the "IGNITION" signal is generated, the LNG pump 4 regulates the temperature in the combustion chamber, and the $\mathrm{O}_{2}$ pump 3 regulates the minimum excess oxygen in the combustion chamber. The water supply to the combustion chamber is blocked by a shutoff valve 27 . The $\mathrm{H}_{2} \mathrm{O}$ pump 1 drives the water in a closed circuit (the $\mathrm{H}_{2} \mathrm{O}$ heat exchanger 12 , the turbine unit cooled by water, and it returns to the collector of a low-pressure condenser). The circulation pumps are switched on and operate in the mode of minimal pumping of water through the circulation circuits. The energy complex is in warm-up mode until the key parts reach operating temperatures. This mode is accompanied by an increase in pressure at the inlet of the compressor, as the working circuit is replenished with fuel combustion products. The part that determines the time of this process is the external power housing of the turbine unit. This is a very massive, thickwalled part. The heating speed of the external power housing of the turbine unit is assumed to be $2 \mathrm{~K} / \mathrm{min}$. The operating temperature of this housing is $400-440 \mathrm{~K}$. Therefore, the warm - up time will be 60-70 minutes. The estimated total heat capacity of heated parts is $30 \mathrm{MJ} / \mathrm{K}$, and the required heat capacity for heating is about $1 \mathrm{MW}$. After power housing of the turbine unit reaches the operating temperature, the compressor 18 operating mode begins to increase and the fuel supply to the combustion chamber increases, so that the temperature of the working fluid at the turbine inlet increases at a predetermined rate. The pressure at the compressor inlet continues to increase, and the pressure at the compressor outlet increases even more intensively (the degree of pressure increase in the compressor increases). When pressure at the compressor outlet exceeds pressure in the $\mathrm{CO}_{2}$ liquefaction unit, the shutoff valves 28 and 30 are opened so that the flow after the compressor enters the high-pressure contact condenser, and from it to the $\mathrm{CO}_{2}$ liquefaction unit. Together with the opening of these valves, the $\mathrm{CO}_{2}$ feed pump 2 is activated. The $\mathrm{CO}_{2}$ feed pump controls the pressure at the turbine inlet. At a certain pressure, the rotor of the turbine block begins to accelerate. The $\mathrm{CO}_{2}$ feed pump controls the acceleration of the turbine block rotor. When it reaches the rated speed, the $\mathrm{CO}_{2}$ pump synchronizes the turbine unit rotor with the power grid and connects the generator to the power grid. After connecting to the power grid, pressure and temperature in front of the turbine continue to increase at the specified speed until the nominal temperature is reached and the power plant's own consumption is fully compensated. From this point in time, the launch is considered complete, and the power unit is ready to accept the required load.

Figure 2 shows a time diagram of changes in the main parameters during the start-up from the cold state. 


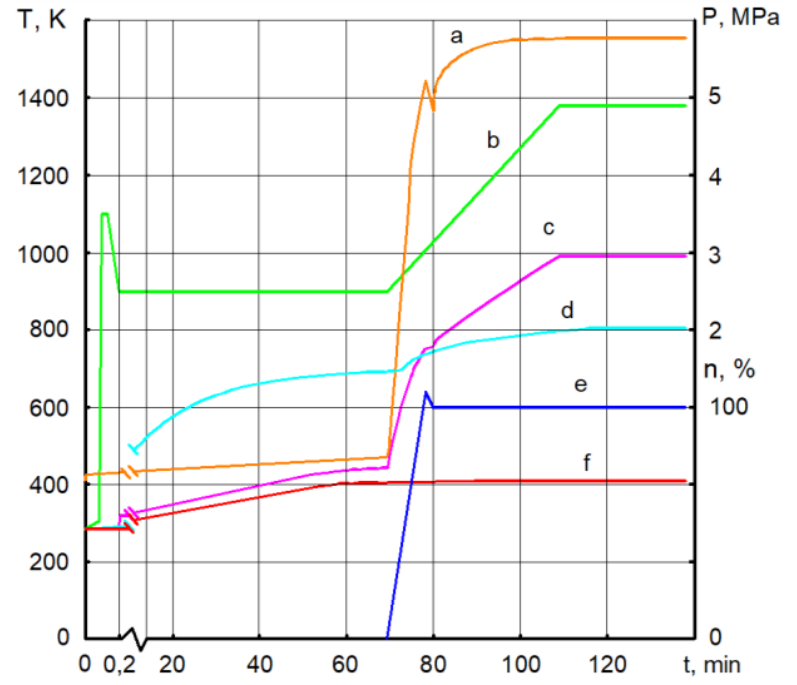

Fig. 2. Time diagram of changes in the main parameters during the start-up from the cold state

$\mathrm{a}$ - pressure of working medium at turbine inlet; $\mathrm{b}$ - temperature of working medium at turbine inlet; $\mathrm{c}$-temperature of the most heated parts of the turbine unit; $d$ - temperature of heat exchangers; e - rotation frequency; $\mathrm{f}$ - temperature of the external power housing of the turbine unit.

The entire process of starting from the cold state is 1.5-2 hours.

\section{Hot start algorithm}

The hot state refers to a situation when the equipment is not cooled down after the previous shutdown. In the hot state, the temperature of the equipment is intermediate between the operating temperature and the ambient temperature. In general, the algorithm for starting from a hot state is almost no different from starting from a cold state, but the warm-up time can be significantly reduced. The closer the temperatures of the determining components (mainly the external power housing of the turbine unit) are to their operating values, the less time it will take to warm up. Fig. 3 shows a time diagram of the change in the main parameters during the start-up from the hot state, when the state of the external power housing of the turbine unit corresponds to the operating temperature.

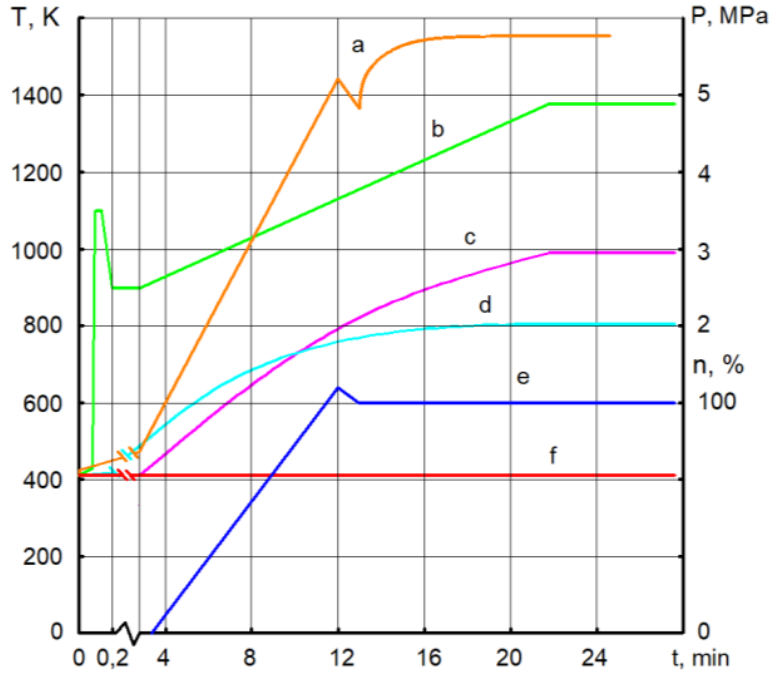

Fig. 3. Time diagram of the change in the main parameters during the start-up from the hot state

$\mathrm{a}$ - pressure of working medium at turbine inlet; $\mathrm{b}$ - temperature of working medium at turbine inlet; $\mathrm{c}$-temperature of the most heated parts of the turbine unit; $d$ - temperature of heat exchangers; e - rotation frequency; $\mathrm{f}$ - temperature of the external power housing of the turbine unit.

In this case, the process from the moment the "START" command is received to the readiness to accept the external load will take 20-25 minutes.

\section{Standby state start algorithm}

One of the CCCGT features is that it is possible to control the pressure in the working circuit. This makes it possible to turn off the energy complex not completely, but to leave it in the standby state. In the standby state, the fuel and oxygen supply is blocked, and the electric generator remains connected to the network. Thus, the electric generator switches to the motor mode and rotates the rotor, overcoming the loss of friction and ventilation. When operating in standby mode, the shutoff valve 29 is closed and the overflow valve 35 is detached. The pressure in the working circuit is lowered to the minimum value, the turbine switches to the compressor mode. The energy spent on the turbine drive is used to compensate for heat losses and, thus, the temperature mode of the hot flow part is kept close to the working one. At the command "START", the shutoff valve 29 opens from the standby state and the overflow valve 35 is closed. The additive $\mathrm{CO}_{2}$ compressor 18 is switched on, and the circulation pumps 14 and 15 are switched on. Feed pumps $1,2,3$ and 4 are switched on, and the ignition system is switched on. The $\mathrm{CO}_{2}$ feed pump 2 increases the pressure at the turbine inlet according to a given time law. The LNG 4 feed pump regulates the temperature at the turbine inlet according to a given time law. The start-up is completed when the temperature of the working fluid reaches the nominal value, and the pressure of the working fluid at the turbine inlet ensures the generation of electricity in an amount sufficient to cover all the needs for the power plant's own needs. Figure 4 shows a time 
diagram of changes in the main parameters during the startup from the standby state.

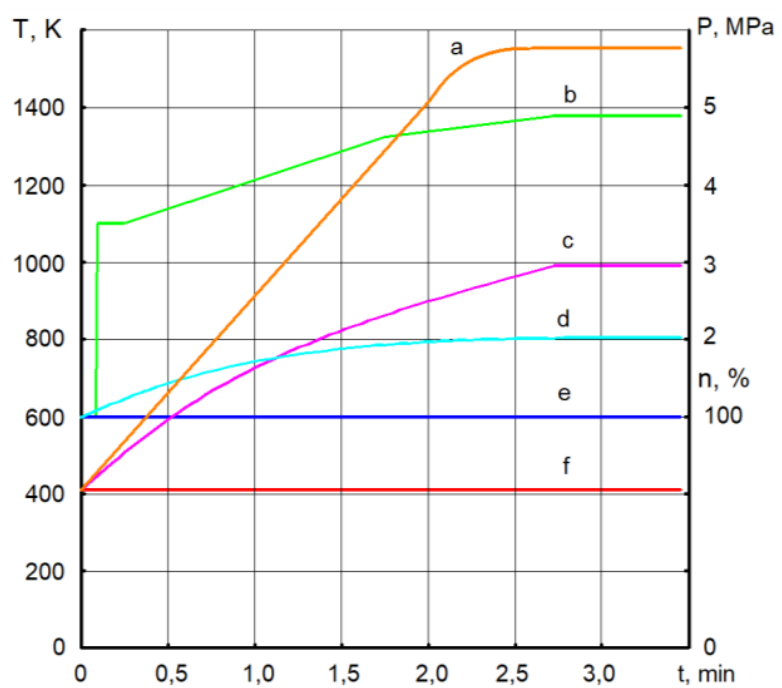

Fig. 4. Time diagram of changes in the main parameters during the startup from the standby state

$a$ - pressure of working medium at turbine inlet; $b$ - temperature of working medium at turbine inlet; $\mathrm{c}$-temperature of the most heated parts of the turbine unit; $d$ - temperature of heat exchangers; e - rotation frequency; $\mathrm{f}$ - temperature of the external power housing of the turbine unit.

In this case, the process from the moment the "START" command is received to the readiness to accept the external load will be 2.5-3 minutes.

\section{Transients from one steady state to another}

The law of regulating the parameters of the CCCGT is proposed such that the constant temperature of the working fluid at the turbine inlet is maintained at all operating modes. In addition, the rotor speed of the turbine block is not constant in all modes. It is envisaged that there will be storage facilities for the initial liquid substances $\left(\mathrm{CO}_{2}, \mathrm{O}_{2}\right.$ and $\left.\mathrm{LNG}\right)$, so that the operation of the fuel infrastructure, the cooling unit for the $\mathrm{CO}_{2}$ liquefaction and the air separation unit will not affect the dynamic characteristics of the energy complex. Of the inertial links that negatively affect the dynamic characteristics of the energy complex, only pumps 1, 2, 3 and 4 should be taken into account. Given the relatively small moments of inertia of the rotors of these pumps, very high dynamic characteristics can be predicted. But, of course, at the subsequent design stages, it will be necessary to pay attention to the dynamic characteristics of such energy complexes.

\section{Normal stop algorithm}

Stopping the CCCGT under normal conditions will consist of the following sequence of actions. All consumers of energy for their own needs are transferred to power from external sources. The pressure in the combustion chamber is reduced to a minimum. The fuel and oxygen supply to the combustion chamber is blocked. The pumps 1, 2, 3, 4 are switched off. The additive compressor 18 decreases the pressure in the working circuit to the value of the standby state. Shutoff valves 27 , $28,29,30,31,32$ are closed, overflow valves 35 and 36 are opened. The circulation pumps 23, 24 are switched off. After that, the energy complex is on standby. The complex can be on standby for an unlimited period of time. But if there is a need to stop the complex completely, then the electric generator is disconnected from the network.

\section{Emergency stop algorithm}

Various emergency situations with different degrees of danger are possible. Depending on the danger degree, different algorithms of the measures are possible. Only such an emergency condition, that requires an emergency stop or preventing the development of an accident, is considered. In such a situation, the fuel and oxygen supply to the combustion chamber is stopped as quickly as possible, for this purpose, shutoff valves 31,32 are closed and the valves that drain the contents of the pipelines into the "candles" 25, 26 are opened. At the same time, pumps 2, 3 and 4 are switched off, the electric generator is disconnected from the mains, shutoff valves 27, 28, 29, 30 are closed, and the overflow valve 36 is opened. The compressor 18 and $t$ circulation pumps 23, 24 are switched off. Depending on the development of the emergency situation, the emergency stop may be completed at this point, or some other measures may be taken. Of course, such an emergency stop entails the release of a certain amount of fuel and carbon dioxide into the atmosphere, but the number of such stops should be kept to a minimum.

\section{Conclusions}

A simplified model of power complexes operating on CCCGT cycle is developed, which makes it possible to study dynamic characteristics of such installations at earliest stages of their creation.

As an example, preliminary studies of the transients of an energy complex with a capacity of $60 \mathrm{MW}$ are carried out.

A variant of the algorithm concept for start/stop and transients of such an energy complex is proposed. This concept includes algorithms for starting from the three initial states of the energy complex (cold, hot, and standby).

The time and energy-consuming characteristics of a launch were evaluated. It is shown that from the cold state, such an energy complex, can be started and brought to the nominal mode in 1.5-2 hours. At the same time, the energy costs will not exceed the energy consumption for its own needs in the nominal mode. The start-up time from the hot state can be reduced to 20-25 minutes, and the start-up from the standby state can be 2.5-3 minutes.

The proposed concept of control algorithms for transient modes is such that the temperature state of main components and parts of the energy complex is provided 
at a constant temperature. Therefore, the time of transients will be determined only by a speed of the regulatory authorities. Consequently, power systems operating on CCCGT cycle can operate equally efficiently, both in the basic mode and in the power control mode.

The research was financially supported by the Russian Science Foundation (project № 19-19-00558).

\section{References}

1. Sanz W., Jericha H., Luckel F., Heitmeir F. A further step towards a Graz cycle power plant for $\mathrm{CO} 2$ capture //ASME Paper GT2005-68456, ASME Turbo Expo. 2005.

2. Oxy-fuel gas turbine, gas generator and reheat combustor technology development and demonstration / R. Anderson, F. Viteri, R. Hollis et. al. //ASME Paper GT2010- 23001, ASME Turbo Expo. 2010.

3. Yang H. J., Kang D.W., Ahn J.H., Kim T.S. Evaluation of design performance of the semi-closed oxy-fuel combustion combined cycle //Journal of Engineering for Gas Turbines and Power. 2012. Vol. 134. No 11. P. 111702.

4. High efficiency and low cost of electricity generation from fossil fuels while eliminating atmospheric emissions, including carbon dioxide / R.J. Allam, M.R. Palmer, G.W. Brown et. al. // Energy Procedia. 2013. Vol. 37. P. 1135- 1149.

5. Andrey Rogalev, Evgeniy Grigoriev, Vladimir Kindra, Nikolay Rogalev Thermodynamic optimization and equipment development for a high efficient fossil fuel power plant with zero emissions // Journal of Cleaner Production 236 (2019), 117592.

6. Kosoi A.S., Popel O.S., Sinkevich M.V. METHOD AND PLANT FOR MECHANICAL AND THERMAL ENERGY GENERATION// Patent RU 2 651918 C1: 24.04.2018 Bull. № 12. http://www1.fips.ru/wps/PA_FipsPub/res/BULLETI N/IZPM/2018/04/27/INDEX RU.HTM

7. A.S. Kosoj, A.A. Kosoj, M.V. Sinkevich, Y.A. Antipov METHOD AND PLANT FOR MECHANICAL AND THERMAL ENERGY GENERATION // Patent RU 2665794 C1: 04.09.2018 Bull. № 25 .

8. The Conceptual Process Arrangement of a Steam-Gas Power Plant with Fully Capturing Carbon Dioxide from Combustion Products / A. S. Kosoi, Yu. A. Zeigarnik, O. S. Popel, M. V. Sinkevich, S. P. Filippov, V. Ya. Shterenberg // Thermal Engineering, 2018, Vol. 65, No. 9, pp. 597-605. DOI: 10.1134/S0040601518090045 A. S. Kosoi, Yu. A. Zeigarnik, O. S. Popel et. al., Thermal Engineering, 65(9), 597-605 (2018)

9. Mikhail Sinkevich, Yuriy Borisov, Nikolay Fominykh, Eldar Ramazanov, Oleg Popel Analysis of the compressorless combined cycle gas turbine unit performance efficiency in district heating systems // E3S Web of Conferences 209, 03008 (2020).

10. Mikhail Sinkevich, Yuriy Borisov, Anatoliy Kosoy, Eldar Ramazanov, Oleg Popel Comparative analysis of the Allam cycle and the cycle of compressorless combined cycle gas turbine unit // E3S Web of Conferences 209, 03023 (2020). 